\title{
Universiteit
}

Leiden

The Netherlands

\section{In situ real-time observation of thin film deposition: roughening, zeno effect, grain boundary crossing barrier, and steering}

Rost, M.J.

\section{Citation}

Rost, M. J. (2007). In situ real-time observation of thin film deposition: roughening, zeno effect, grain boundary crossing barrier, and steering. Physical Review Letters, 99, 266101. doi:10.1103/PhysRevLett.99.266101

Version: $\quad$ Not Applicable (or Unknown)

License: $\quad$ Leiden University Non-exclusive license

Downloaded from: https://hdl.handle.net/1887/61288

Note: To cite this publication please use the final published version (if applicable). 


\title{
In Situ Real-Time Observation of Thin Film Deposition: Roughening, Zeno Effect, Grain Boundary Crossing Barrier, and Steering
}

\author{
M. J. Rost \\ Kamerlingh Onnes Laboratory, Leiden University, P.O. Box 9504, 2300 RA Leiden, The Netherlands \\ (Received 22 June 2007; published 27 December 2007)

\begin{abstract}
We report on the first atomic-scale real-time in situ investigation of the growth of a polycrystalline gold film during its deposition performed with a scanning tunneling microscope. Continuously scanning while depositing the film enables the direct observation of atomic processes. The grain boundaries play a crucial role in the evolving film structure, as they initiate mound formation, thereby significantly increasing the film roughness. A possible additional roughness increase comes from atom steering, which also can delay the film closure in the early stages during film growth.
\end{abstract}

DOI: 10.1103/PhysRevLett.99.266101

Thin polycrystalline metal films are becoming increasingly important, as is reflected in the multitude of applications in different fields [1], e.g., nanotechnology, nanooptics, microelectronics, and catalysis. The intrinsic link between the film properties and the precise film structure enables the production of films with tuned properties. Ideally one would like to control the texture, grain boundary (GB) orientation, grain size distribution, adhesion, intrinsic stress, and the overall morphology down to the nanometer scale. The great interest in the dependence between the developing film structure and the precise deposition parameters resulted in the establishment of the well-known structure zone models [2-7] that summarize the current background knowledge. However, these phenomenological models certainly lack information on the atomistic details and processes that take place during thin film growth. The continuous miniaturization of thin film applications clearly demands fundamental research that links well-known atomic processes, such as diffusion and nucleation, with the mesoscopic evolution of film properties, both during film growth as well as during a postdeposition treatment, e.g., heating, applying stress, and additional coating. A typical example is given by the evolution of the intrinsic stress of thin films during and after the deposition [8-13]. On growth interruption one observes significant changes of the film stress, which must be linked to morphological changes. However, the underlying atomic processes are still under debate. In order to unravel such processes we need an in situ real-time technique that is nondestructive, surface sensitive, and capable of obtaining atomic resolution. In an earlier paper [14] we reported on the post-deposition anneal treatment of a polycrystalline film in which we observed grain growth.

In this Letter we show that it is even possible to observe the growth of a thin polycrystalline metal film with a scanning tunneling microscope (STM) during its deposition. We are continuously scanning with atomic step resolution while depositing significant thicknesses of tens of monolayers. Analyzing our observation, we show that the GBs play a crucial role in the evolving film structure. The
PACS numbers: 81.15.Aa, 68.35.Ct, 68.37.Ef, 68.55.Ac

GBs naturally initiate mound formation if an EhrlichSchwoebel barrier exists at the steps that are part of the grain surfaces. As a consequence the overall roughness increases during film growth and the initial equilibrium shape of a grain evolves into a moundlike structure. On single crystalline surfaces four different growth regimes are well established, depending on the deposition flux and substrate temperature: step-flow growth, layer-by-layer growth (birth and spread), mound formation, and selfaffine growth [15]. Although we clearly observe pure step-flow growth on top of the individual, single crystalline

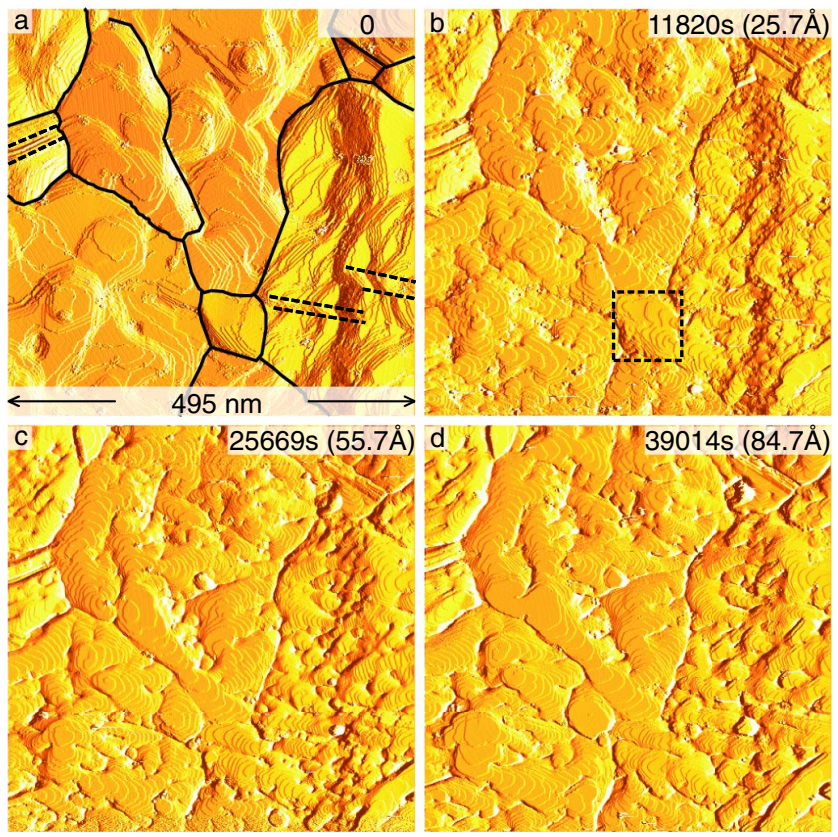

FIG. 1 (color online). STM images taken from a movie that was recorded during the deposition of a polycrystalline Au film at $293 \mathrm{~K}$ while continuously scanning. We used a differential filter to enhance the step contrast and indicate the time of recording and film thickness in the upper right corners. GBs are denoted with black lines in (a); dashed lines indicate twin boundaries. Step meanders are visible in the box in (b). 
grains, we simultaneously find mound formation initiated by the GBs. In general, GBs can be transparent for surface diffusing atoms, but mostly we expect them to introduce a diffusion barrier, which we call the GB crossing barrier. Both this barrier and the initial equilibrium grain shape with a groove at the root of the GB cause a termination of the flowing steps at the position of the GBs. This effect leads to mound formation, thereby increasing the overall film roughness.

The experiments are performed with a home-built STM described in [16]. In order to ensure a well-defined starting situation, we evaporated a $30 \mathrm{~nm}$ thick gold film in situ in our ultrahigh vacuum chamber on a quartz substrate as described in [14]. Immediately after deposition, the sample was first transferred to the STM without breaking the vacuum and then annealed at $T=750 \mathrm{~K}$, resulting in a well characterized gold film: highly (111) textured with an average grain size of $\sim 250 \mathrm{~nm}$ [14].

Figure 1(a) shows the starting situation prior to the deposition experiment. From a careful analysis of all terrace slopes we can determine the tilts, which provide us with first information on the orientation of the individual grains. Because of our new control electronics [17], we resolve not only the macroscopic polycrystalline surface morphology, but simultaneously we receive atomic information on the individual terraces: the well-known Herringbone reconstruction of $\mathrm{Au}(111)$ [18]. The modest height variation of this reconstruction is vaguely visible as parallel lines on the terraces in Fig. 1(a). From this surface reconstruction we extract information on the twist of the individual grains. Using both the tilt and the twist data, we can pinpoint the positions of the GBs indicated with the black lines in Fig. 1(a). Figures 1(b)-1(d) show the film evolution during the deposition at times $t=11820,25669$, and $39014 \mathrm{~s}$, respectively. The complete movie is available as supplemental material [19]. Limited by the geometry of the current setup, the film was deposited at $293 \mathrm{~K}$ with a flux equivalent of $7.8 \AA$ per hour using $99.999 \%$ pure gold. During deposition the background pressure of the chamber was less than $8 \times 10^{-10}$ mbar. All STM images were acquired at a sample voltage of $-0.7 \mathrm{~V}$ and at tunneling currents below $0.1 \mathrm{nA}$.

Inspecting the movie [19] we extract several observations. The total roughness of the film increases as the number of open atom layers increases during deposition. The steps are flowing over the surface until they reach the positions of the GBs, where they usually come to rest. As a consequence the GBs stay at their initial positions. The highest point of a grain is usually formed by a twodimensional island, on top of which we observe the nucleation of the next layer when growth proceeds. We also find an exception in the lower left part of the movie, where a dislocation nucleates and evolves into two growing spirals during deposition. The initial compact steps evolve into step meanders [see box in Fig. 1(b)]. Comparing our observations with the film structure zone models [2,3], we assign our film growth to zone 2 (columnar growth).
In order to quantify the roughness increase and assess its characteristic length scale, we start analyzing our data by calculating the second moment of the height-difference correlation function

$$
C_{2}(x)=\left\langle\left|h\left(x_{i}+x\right)-h\left(x_{i}\right)\right|^{2}\right\rangle^{1 / 2}
$$

for each individual image, where $h\left(x_{i}\right)$ denotes the height of pixel number $i$, and $x$ the correlation distance. Plotting $C_{2}(x)$ versus $x$ in a double logarithmic plot [see Fig. 2(c)], we extract the interface width $\xi_{\perp}$ and the parallel correlation length $\xi_{\|}$from the kink position. The former parameter describes the roughness amplitude of the film, whereas the latter describes the in-plane length scale one has to bridge in order to reach the related film roughness. The result of this analysis is shown in Figs. 2(a) and 2(b). It is striking that the roughness increases linearly with time, whereas the parallel correlation length stays constant within its fluctuations. This behavior indicates the existence of a growth instability, as it substantially differs from the standard scaling theory [20] that describes the relationship between these two values in time: an increase in one of the quantities usually goes hand in hand with an increase in the other. Where is the increase in roughness coming from? One might consider single crystalline effects on top of the individual grains as well as effects related to the polycrystalline network with its GBs. Information comes from the average parallel correlation length $\xi_{\|}=50 \mathrm{~nm}$. It can be shown that the determination of this value from the kink position underestimates the real correlation length with a factor of $\sim 2$ [21]. Therefore the dominating roughness develops on a length scale of $\sim 100 \mathrm{~nm}$, which fits well with the average grain radius of $\sim 125 \mathrm{~nm}$ (see [14]). In the following we consider effects that increase the roughness on the length scale of the grain size.

As mentioned above we clearly observe the nucleation of the next layer in the form of a new two-dimensional island on the topmost island of each of the individual grains. Simultaneously we observe the termination of the flowing steps at the positions of the GBs. The combination of both effects leads to an increase in roughness if atoms

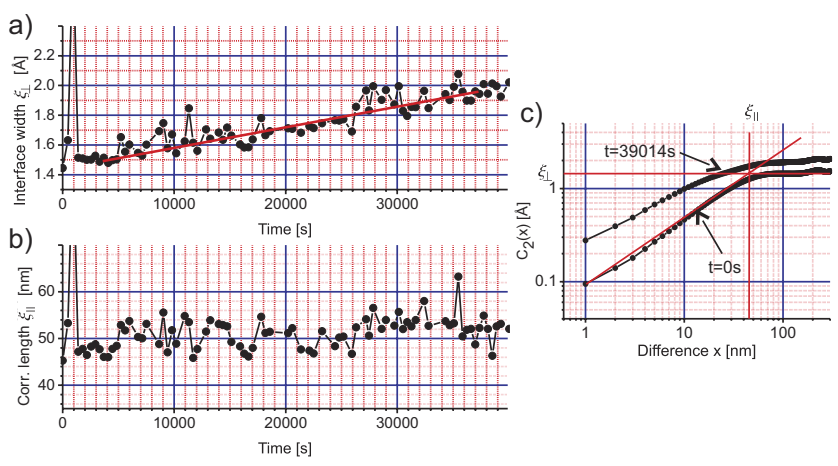

FIG. 2 (color online). Correlation values during film growth: (a) interface width $\xi_{\perp}$, (b) parallel correlation length $\xi_{\|}$, and (c) two examples of the height-difference correlation function; from the kink position we determine $\xi_{\perp}$ and $\xi_{\|}$. 
face an additional barrier for the process of diffusing down over a descending step (Ehrlich-Schwoebel barrier $[22,23])$. This barrier leads to a so-called uphill current, as depositing atoms on a terrace preferentially attach to the ascending step via diffusion. As a consequence the speed of a flowing step scales with the width of the lower terrace. Figure 3(a) shows the development of an equally spaced array of steps under growth conditions. We assume a hard barrier, e.g., a GB at the right-hand side of the image, as well as constant nucleation of new islands. When the steps approach the barrier, their lower terrace gets shorter, which results in a decrease of their speed. This leads to mound formation, similar to the mound formation observed on single-crystal surfaces (often referred to as the Zeno effect [24,25]). However, on single-crystal surfaces, mound formation starts only after the creation of surface parts with a positive curvature, which requires the nucleation of $2 \mathrm{D}$ islands on the initial flat terraces. This initial nucleation is not necessary here. The GBs, which separate the grains, predefine not only barriers but also a moundlike structure, because the surface initially adopts a domelike equilibrium shape between neighboring GBs [14]. This shape usually implies that the initial terrace widths are shorter the closer a terrace is located to the root of a GB. In this case mound formation occurs naturally. Atom incorporation into the GBs [26,27], might affect mound formation, especially in the beginning, when the film still deviates from its steadystate compressive stress during growth.

We can verify the mechanism described by analyzing both the local out-of-plane growth and the evolving shape of a grain. Figure 3(b) shows the local variation of the average growth at $39014 \mathrm{~s}$ : darker shades indicate less growth than average. The local growth $G_{t}(x, y)$ is calculated from the absolute height $h_{t}(x, y)$ of an image at time $t$ :

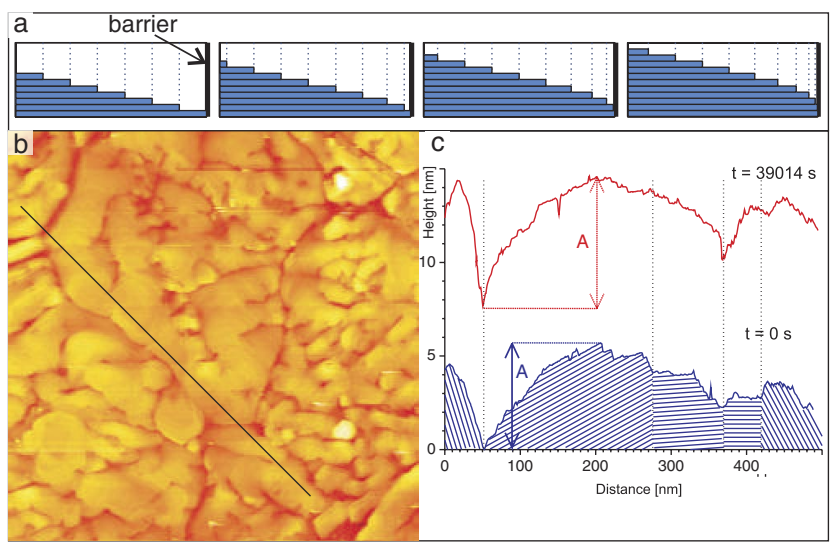

FIG. 3 (color online). (a) Schematic drawing of the mound formation during growth: equally spaced steps approach a barrier, e.g., a GB, which is located at the right side of the image. (b) Local variation of the average growth for $t=39014 \mathrm{sec}$. (please note that this is not an STM height image) (c) Surface height profiles at the position of the black line in (b); GBs, indicated with dashed lines, get steeper and grains evolve to mounds. One exception is also visible.

$$
G_{t}(x, y)=\left[h_{t}(x, y)-h_{t=0}(x, y)\right]-\text { const. }
$$

The GBs are clearly visible, which indicates significantly less local growth than the average growth of the entire film. To further elucidate the general mechanism of roughness increase, we compare the grain shapes. Figure 3(c) shows two height lines at the same location at times $t=0$ and $t=$ $39014 \mathrm{~s}$. The positions of the GBs are indicated with dashed lines. During growth the GBs get steeper, the lateral positions of the boundaries remain almost the same, the vertical positions of the roots shift upward, the grain shapes get more moundlike, and the total surface width, $A$, increases. The height increase of the roots indicates a downhill current of atoms, e.g., via downhill funneling [28]. Please note also the two exceptions (at $275 \mathrm{~nm}$ and $420 \mathrm{~nm} ; t=0 \mathrm{~s}$ ) to the naïve case of two grains connected to a boundary exclusively via downward steps from both sides. The $\{111\}$ crystal planes of the individual grains are indicated with the hatching. We observe such exceptions in cases of combinations of (a) large surface slopes, (b) strong GB inclination, (c) grain (mis)orientation, and (d) small GB energy (i.e., large grooving angle, like low angle, or twin boundaries). The GB at $275 \mathrm{~nm}$ overgrows as expected on purely geometric arguments, whereas the GB at $420 \mathrm{~nm}$ steepens. The different behavior indicates the importance of a GB crossing barrier. Without this barrier the GBs should get overgrown, as the steps capture the atoms from the flat terrace of the other grain. If an adatom GB crossing barrier exists that makes the diffusion of adatoms from one grain to the other more difficult than terrace diffusion, mound formation will also occur for these special GBs. In general the grains evolve to moundlike structures, which leads to an increase of the roughness. This conclusion also explains the nearly constant parallel correlation length: the morphological change affects only the individual grains and not the GB network.

An additional contribution to the roughness might come from some process that takes place on the individual grains and is not related to the GBs. Such a process should also be observable during the growth of pure single crystals. When inspecting the images during growth, one notices evolving step meanders. These structures remind one of the wellknown homoepitaxial step-flow instability [29], which would also lead to an increase in roughness. However, if the major contribution of the roughness would stem from these structures that develop during the deposition on top of the individual grains, the correlation length should decrease instead of being constant. For small distances the step meanders clearly lead to a roughness increase; see Fig. 2(c). Taking into account both this increase and the corresponding slope change for distances that are smaller than $\xi_{\|}$, we estimate the contribution of the step meanders to the total roughness increase at maximum to $10 \%$. Additional proof comes from the surface width: the amplitude, $A$, of the grain shapes. On average this amplitude increases with a factor of $\sim 1.4$ during deposition [see 


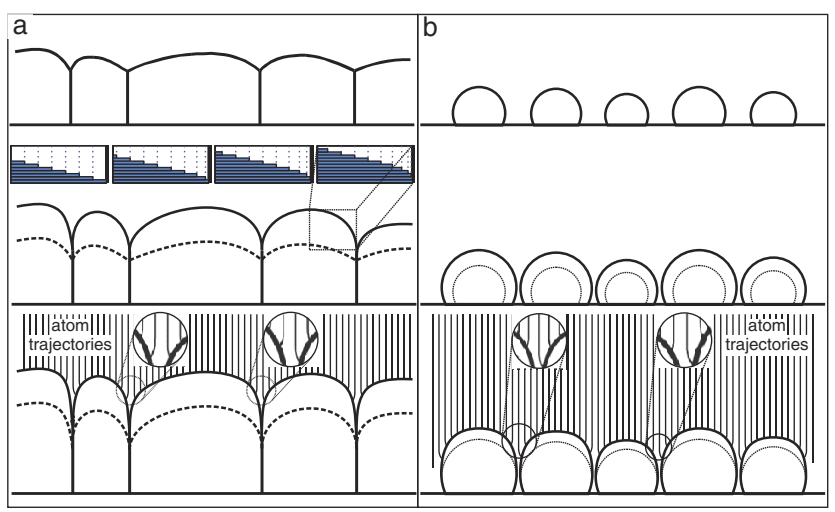

FIG. 4 (color online). Schematics of growth, due to Zeno, GB crossing barrier, and steering effects (the vertical lines in the lowest panels denote atom trajectories). (a) Roughening and grain separation during polycrystalline film growth. (b) Delay of the film closure after nucleation.

Fig. 3(c)], which is in good agreement with the ratio of the total roughnesses of 2.02/1.45 [see Fig. 2(a)].

Finally we want to point out a second mechanism that results in a further steepening of the GBs during growth. It is known that the trajectories of depositing atoms get deflected toward the surface, due to atom-surface interactions. This so-called steering influences the growth and can, together with shadowing, lead to dramatic changes in the evolving surface structure. This has been verified on single crystals [30]. On polycrystalline surfaces we realize that the combination of the steepening of the GBs due to mound formation, together with steering, leads to interesting additional phenomena. In the absence of downhill currents, grains separate during the growth: the out-ofplane growth speed of the root of the GBs decreases with increasing film thickness until it eventually becomes zero. The growth at the root stops when the surface steepness is so large that all incoming atoms are steered to the sides, away from the root. We would like to point out that this is even valid for evaporation at normal incidence. In the case of a nonzero angle of incidence shadowing also becomes important. Even in the presence of downhill currents and the incorporation of atoms in the GBs, kinetic limitations will make the out-of-plane growth speed at the roots significantly lower than that in the middle of the grains [sketched in Fig. 4(a)]. Surface diffusion, driven by curvature, will eventually result in an equilibrium growth shape.

We expect that effects similar to the grain separation play an important role in the growth of a polycrystalline film with a contact angle larger than $90^{\circ}$ during its stages from nucleation of the individual single crystals until film closure [see Fig. 4(b)]. After nucleation the individual crystals (grains) grow until they almost touch their neighbors. Eventually the intergrain distance becomes so small that all atoms from the evaporation beam between two crystals are steered to the sides. Similar to the above description, the grains grow higher without the immediate formation of a GB. As a consequence the film closure is delayed. We expect this phenomenon to be of importance at low temperatures or high deposition rates, next to the effect that is suggested in [31] and derived from an elegant study of the early stages of a polycrystalline Ag film grown on amorphous $\mathrm{Si}$.

In closure, we expect that our findings are of a very general nature and that the processes described will occur on almost every polycrystalline film during both its nucleation and growth.

I kindly acknowledge J.W.M. Frenken for the support of this project with his microscope, as well as V. Fokkema for helpful discussions.

[1] C. K. Hu et al., Mater. Chem. Phys. 52, 5 (1998).

[2] B.A. Movchan and A. V. Demchishin, Fiz. Met. Metalloved. 28, 83 (1969).

[3] J. A. Thornton, Annu. Rev. Mater. Sci. 7, 239 (1977).

[4] R. Messier et al., J. Vac. Sci. Technol. A 2, 500 (1984).

[5] P. B. Barna et al., Thin Solid Films 317, 27 (1998).

[6] C. V. Thompson, Annu. Rev. Mater. Sci. 30, 159 (2000).

[7] I. Petrov et al., J. Vac. Sci. Technol. A 21, S117 (2003).

[8] A. L. Shull et al., J. Appl. Phys. 80, 6243 (1996).

[9] E. Chason et al., Phys. Rev. Lett. 88, 156103 (2002).

[10] F. Spaepen, Acta Mater. 48, 31 (2000).

[11] C. Friesen and C. V. Thompson, Phys. Rev. Lett. 93, 056104 (2004).

[12] R. Koch, D. Hu, and A. K. Das, Phys. Rev. Lett. 94, 146101 (2005).

[13] C. Friesen and C. V. Thompson, Phys. Rev. Lett. 95, 229601 (2005).

[14] M. J. Rost, D. A. Quist, and J. W. M. Frenken, Phys. Rev. Lett. 91, 026101 (2003).

[15] T. Michely and J. Krug, Islands, Mounds, and Atoms (Springer, New York, 2004).

[16] M.S. Hoogeman et al., Rev. Sci. Instrum. 69, 2072 (1998).

[17] M. J. Rost et al., Rev. Sci. Instrum. 76, 053710 (2005).

[18] U. Harten et al., Phys. Rev. Lett. 54, 2619 (1985), and references therein.

[19] See EPAPS Document No. E-PRLTAO-99-006751 for an STM movie of the deposition of a polycrystalline $\mathrm{Au}$ film. We are continuously scanning while depositing the film. For more information on EPAPS, see http:// www.aip.org/pubservs/epaps.html.

[20] P. Meakin, Fractals, Scaling and Growth Far from Equilibrium (Cambridge University Press, Cambridge, 1998).

[21] M. J. Rost (to be published).

[22] G. Ehrlich et al., J. Chem. Phys. 44, 1039 (1966).

[23] R. L. Schwoebel et al., J. Appl. Phys. 37, 3682 (1966).

[24] I. Elkinani et al., J. Phys. I (France) 4, 949 (1994).

[25] M. Kalff et al., Surf. Sci. 426, L447 (1999).

[26] C.-W. Pao et al., Phys. Rev. Lett. 99, 036102 (2007).

[27] J.S. Tello et al., Phys. Rev. Lett. 98, 216104 (2007).

[28] H.-J. Ernst et al., Phys. Rev. Lett. 72, 112 (1994).

[29] T. Maroutian, L. Douillard, and H. J. Ernst, Phys. Rev. Lett. 83, 4353 (1999).

[30] S. van Dijken, L. C. Jorritsma, and B. Poelsema, Phys. Rev. Lett. 82, 4038 (1999).

[31] C. Polop et al., New J. Phys. 9, 74 (2007). 